SLAC-PUB-11251

May 2005

\title{
Computer Algorithm for Longitudinal Single Bunch Stability Study in a Storage Ring
}

\author{
Sasha Novokhatski \\ Stanford Linear Accelerator Center, Stanford University, Stanford, California 94309
}

\begin{abstract}
We propose a new method for simulation study of the nonlinear interaction of a single bunch and accelerator vacuum chamber elements. We numerically solve the Fokker-Planck time-domain equation for the phase-space distribution function. Original implicit finite-difference scheme is used. The method is very stable and free of the "numerical" diffusion, distortion, or modulation. We introduce quasi-Green function to describe the wake field potentials of bunches of any shape. This allows to get high definition resolution of the bunch particle distribution. We present results and comparison for different kind of instabilities.
\end{abstract}

${ }^{*}$ Work supported by Department of Energy contract DE-AC02-76SF00515. 


\section{INTRODUCTION}

The main effect, which determines the stability of the beam in a storage ring, is electromagnetic interaction of the beam particles with vacuum chamber elements. We describe this interaction by the wake potentials of all vacuum elements in the ring. Phasespace distribution function effectively describes the motion of particles in the bunch. This

function is the solution of the Fokker-Planck equation ${ }^{1}$. We designed an effective numerical algorithm to solve this non-linear equation together with wake field potentials. First results of application of this method to the TESLA storage ring were presented at the TESLA Meeting ${ }^{2}$ in 1998 and next year at the Workshop on Linear Colliders ${ }^{3}$ LC'$^{\prime} 99$. Results for saw-tooth instability for resonator wake potential were presented at EPAC'2000 ${ }^{4}$

\section{WAKE FUNCTION AND QUASI-GREEN FUNCTION}

We describe wake potential $W(s)$ as convolution of charge density distribution $\rho(s)$ and wake function $w(s)$

$$
W(s)=\int_{-\infty}^{s} w\left(s-s^{\prime}\right) \rho\left(s^{\prime}\right) d s^{\prime}
$$

There are only few known analytical expressions for the wake (or Green) function ${ }^{5}$. Green function is the wake potential of a point charge for a particular accelerator element. Direct numerical solution of the Maxwell equations gives wake potentials only for finite length bunches. There are some methods ${ }^{6,7}$ which use wake potential of a short Gaussian bunch as an approximate Green function. Naturally, application of these methods is limited by the minimum bunch length available in the wake field calculations. 
We suggest a new method for the wake potential calculation. We introduce a new wake function $\widetilde{w}=\widetilde{w}\left(s_{q}, s\right)$, which has an additional distance parameter $s_{q}$. We define this function as a "quasi-Green function". With this function we calculate directly (analogue to the formula (1)) the approximation $\widetilde{W}(s)$ of the wake potential

$$
\tilde{W}(s)=\int_{0}^{\infty} \tilde{w}\left(s_{q}, s^{\prime}\right) \rho\left(s+s_{q}-s^{\prime}\right) d s^{\prime}
$$

From the expression (2) one can see that the quasi Green function becomes real Green function, when the distance parameter takes zero value

$$
\tilde{w}\left(s_{q}, s\right) \rightarrow w(s) \text { when } s_{q} \rightarrow 0
$$

Quasi-Green function approaches the bunch wake potential (shifted in distance by $s_{q}$ ), when the distance parameter is larger that the bunch length

$$
\tilde{w}\left(s_{q}, s\right) \rightarrow \tilde{W}\left(s-s_{q}\right) \approx W\left(s-s_{q}\right) \text { when } s_{q} \gg \sigma
$$

So the distance parameter of the quasi-Green function determines the quality of the approximation of the real Green function: smaller parameter - better approximation. Expression (4) gives the simple way for the evaluation of quasi-Green function from the known wake potential. We can find quasi-Green from the requirement of the minimum difference between potentials $\widetilde{W}(s)$ and $W(s)$. For this purpose we vary quasi-green function to give minimum value of the functional:

$$
\min _{\tilde{w}}\left\{\int_{s_{\min }\left(s_{q}\right)}^{s_{\max }}\left(W(s)-\int_{0}^{\infty} \tilde{w}\left(s_{q}, s^{\prime}\right) \rho\left(s+s_{q}-s^{\prime}\right) d s^{\prime}\right)^{2} d s\right\}
$$

at the optimum value of the distance parameter $s_{q}$, which is determined by the quality, and resolution of the given wake potential $W(s)$. If the quality of the wake is good (small 
numerical noise, good agreement with principle of causality) the parameter may be very small. For wake potentials, calculated using code "NOVO", the value of the distance parameter is usually in the region of 3-5 bunch length. For the wake potentials, calculated by the well known codes like $\mathrm{ABCI}$ and MAFIA the optimum value of the distance parameter is considerably larger.

We will demonstrate the efficiency of the method (5) on the example of reconstruction of the wake potentials for a "long" cavity in the vacuum chamber (Fig. 1). To make comparison, we first calculated wake potentials $W(s)$ (using code NOVO) for the different bunch lengths: $0.5,1.0,2.9$ and $20 \mathrm{~mm}$. Then we solved numerically the problem (5) for the wake potential of $1.0 \mathrm{~mm}$ bunch and found quasi-Green function $\tilde{w}\left(s_{q}, s\right)$. Finally, we reconstructed the wake potential $\widetilde{W}(s)$ for $1.0 \mathrm{~mm}$ bunch (same bunch length) with the help of the derived quasi-Green function using formula (2). Both wake potentials are shown in Fig.1: primary wake potential is presented by a red solid line and reconstructed wake potential is shown by light blue circles. One can see perfect agreement.

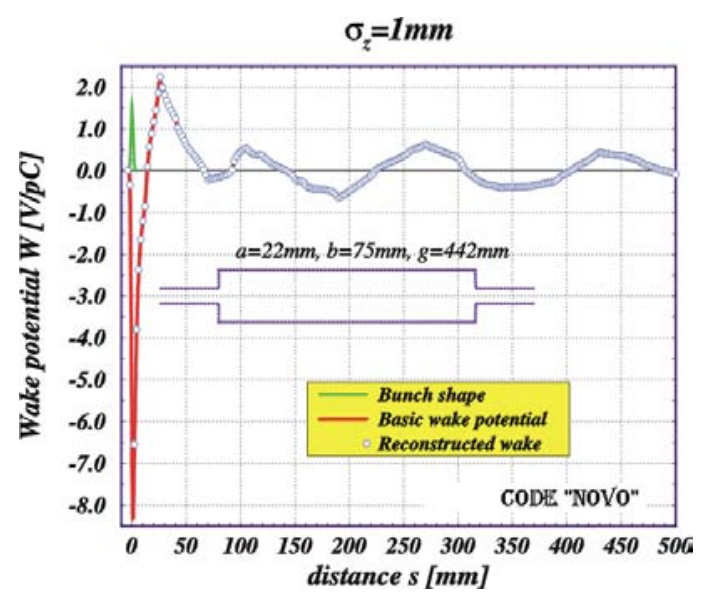

Figure 1: Primary wake potential (red solid line) of a "long" cavity for $1.0 \mathrm{~mm}$ bunch and reconstructed wake potential (light blue circles). Green line shoes bunch shape. 
We also reconstructed the wake potentials of bunches of $2.0 \mathrm{~mm}$ and $20 \mathrm{~mm}$ length using derived quasi-Green function. The results are presented in Fig.2 (left and right plot). One can note very good agreement between calculated and reconstructed wake potentials for different bunch lengths.
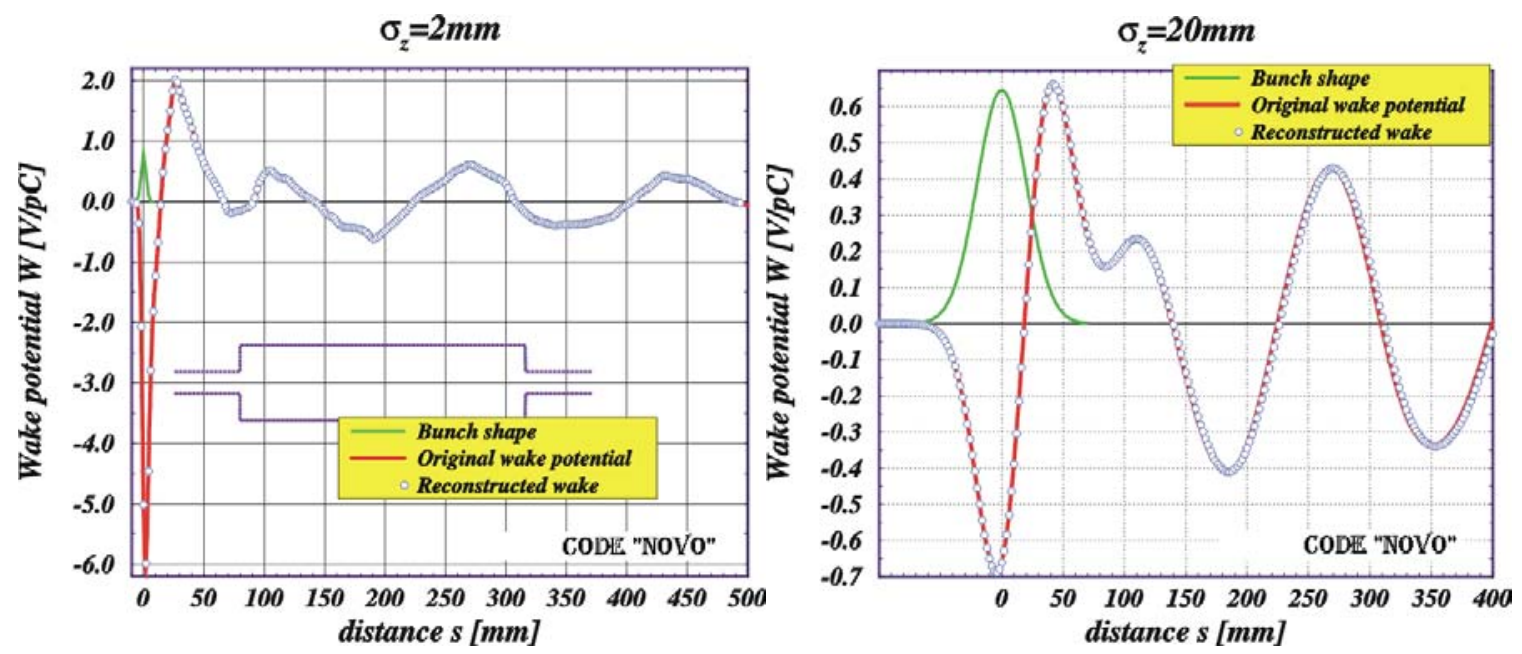

Figure 2: Calculated (solid red line) and reconstructed (light blue circles) wake potentials of $2.0 \mathrm{~mm}$ and $20 \mathrm{~mm}$ bunch. Green line shows bunch shape.

To find the high frequency limit of the derived quasi-Green function we have reconstructed the wake potential for the bunch of two time's smaller length $(0.5 \mathrm{~mm})$. We get good agreement (Fig.4) again. So the presented method allows to reconstruct the wake potential of shorter bunches than we use for evaluation of the quasi-Green function. The frequency limit is determined by the derived quasi Green function. One can see that quasi-Green function (presented in Fig. 3 by blue solid line) has approximately the shape of the wake potential of $0.5 \mathrm{~mm}$ bunch, but shifted by distance parameter. In this case quasi-Green unction allows reconstructing the wake potentials of two times shorter bunches. 


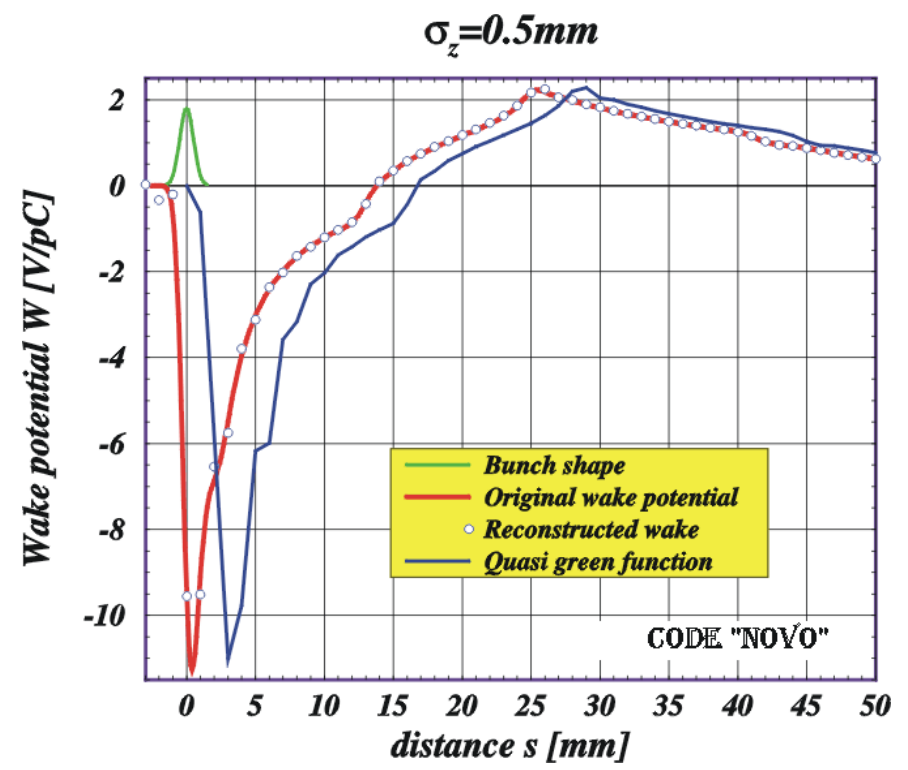

Figure 3: Real (solid red line) and reconstructed (light blue circles) wake potentials of $0.5 \mathrm{~mm}$ bunch. Quasi-Green function is shown by blue solid line. Bunch shape is shown by green line.

\section{FOKKER-PLANCK EQUATION}

To study the effect of the wake fields on the longitudinal beam dynamics in a storage ring we use the solutions of the Fokker-Planck equation for the phase-space distribution function $\psi=\psi(t, x, p)$ of momentum and coordinate

$$
\frac{\partial}{\partial t} \psi+\dot{x} \frac{\partial}{\partial x} \psi+\dot{p} \frac{\partial}{\partial p} \psi=\lambda \frac{\partial}{\partial p}\left\{p \psi+\frac{\partial}{\partial p} \psi\right\}
$$

Time derivatives of the canonical coordinates are:

$$
\begin{aligned}
& \dot{x}=p \\
& \dot{p}=F=-\frac{\sin \left(k_{r f} x+\varphi_{0}\right)-\sin \varphi_{0}}{k_{r f}}+\frac{e N c}{V_{r f} \omega_{r f} \sigma_{0}} W(t, x) \\
& k_{r f}=\frac{\omega_{r f}}{c \sigma_{0}} \quad \lambda=\frac{2}{f_{s} \tau_{\text {damp }}}
\end{aligned}
$$

Bunch density is 


$$
\rho(t, x)=\int_{-\infty}^{\infty} \psi(t, x, p) d p
$$

Wake potential is:

$$
W(t, x)=\int_{-\infty}^{x+x_{q}} \rho\left(t, x^{\prime}\right) \widetilde{w}\left(x_{q}, x+x_{q}-x^{\prime}\right) d x^{\prime}
$$

The coordinate and momentum are normalized by natural (zero-current) value of the bunch length $\sigma_{0}$ and momentum spread $p_{0}$. Time is measured in synchrotron periods. So for the description of the ring we need only:

- $\quad$ natural bunch length $\sigma_{0}$

- bunch charge $e N$

- RF voltage $V_{r f}$

- $\quad$ RF frequency $\omega_{r f}$

- $\quad$ synchrotron frequency $f_{s}$

- damping time $\tau_{\text {damp }}$

- quasi-Green function for wake potential calculation

\section{NUMERICAL METHOD}

For the solution of the Fokker-Planck equation (6) we use the numerical method, based on the two step implicit finite-difference approximation of fourth order: 


$$
\begin{aligned}
& \varphi_{i, j}(1+\gamma)+\varphi_{i+1, j}\left(\frac{1}{4}+\tilde{F}_{j}-\tilde{p}_{i+1}-\frac{\gamma}{2}\right)+\varphi_{i-1, j}\left(\frac{1}{4}-\tilde{F}_{j}+\tilde{p}_{i-1}-\frac{\gamma}{2}\right)= \\
& \psi_{i, j}^{n}(1-\gamma)+\psi_{i+1, j}^{n}\left(\frac{1}{4}-\tilde{F}_{j}+\tilde{p}_{i+1}+\frac{\gamma}{2}\right)+\psi_{i-1, j}^{n}\left(\frac{1}{4}+\tilde{F}_{j}-\tilde{p}_{i-1}+\frac{\gamma}{2}\right) \\
& \tilde{p}_{i}=\frac{3}{8} \lambda p_{i} \frac{\Delta t}{\Delta p} \quad \tilde{F}_{j}=\frac{3}{8} F\left(x_{j}, t\right) \frac{\Delta t}{\Delta p} \quad \gamma=\frac{3}{8} \lambda \frac{\Delta t}{(\Delta p)^{2}} \\
& \psi_{i, j}^{n+1}+\psi_{i, j+1}^{n+1}\left(\frac{1}{4}+\frac{3}{8} p_{i} \frac{\Delta t}{\Delta x}\right)+\psi_{i, j-1}^{n+1}\left(\frac{1}{4}-\frac{3}{8} p_{i} \frac{\Delta t}{\Delta x}\right)= \\
& \varphi_{i, j}+\varphi_{i, j+1}\left(\frac{1}{4}-\frac{3}{8} p_{i} \frac{\Delta t}{\Delta x}\right)+\varphi_{i, j-1}\left(\frac{1}{4}+\frac{3}{8} p_{i} \frac{\Delta t}{\Delta x}\right)
\end{aligned}
$$

Fourier analysis shows that this implicit scheme gives perfect dispersion relation between frequency $\omega_{p}$ and wave vector $k_{p}$ in the phase space for any normalized momentum $p$ (or force $F$ )

$$
\operatorname{tg}\left(\frac{\omega_{p} \Delta t}{2}\right)=p \frac{3}{4} \frac{\Delta t}{\Delta x} \frac{\sin \left(k_{p} \Delta x\right)}{1+0.5 \cos \left(k_{p} \Delta x\right)}
$$

When $k_{p} \Delta x \leq 1$ we have exact relation $\omega_{p}=p k_{p}$, even for critical wave number (of mesh size) $k_{p} \Delta x=\pi / 2$ we still have good relation $\omega_{p}=\frac{3}{\pi} p k_{p}$. It means that our scheme does not produce any numerical diffusion, distortion or modulation. Equations (7) contain three diagonal matrixes, so we can use round-trip algorithm for each time step. For boundary conditions, we use the case when no new particles come to the defined region $\left\{x_{\min } p_{\min } ; x_{\max } p_{\max }\right\}$

$$
\begin{aligned}
& \psi\left(x_{\min }, p\right)=0 \text { when } p>0 \\
& \psi\left(x_{\max }, p\right)=0 \text { when } p<0 \\
& \psi\left(x, p_{\min }\right)=0 \text { when } F>0 \\
& \psi\left(x, p_{\max }\right)=0 \text { when } F<0
\end{aligned}
$$




\section{THE ALGORITHM CHECK}

We have checked the algorithm for the case of a resonator wake function

$$
\begin{aligned}
& w(s)=-\frac{I}{k_{Q}} \frac{d}{d s}\left[\exp \left(-\frac{k}{2 Q} s\right) \sin k_{Q} s\right] \\
& k_{Q}=k \sqrt{1-1 / 4 Q^{2}}
\end{aligned}
$$

We use dimensionless intensity parameter $I$ for description of the ring and resonator wake amplitude $W_{r}$

$$
I=\frac{e N c}{V_{r f} \omega_{r f} \sigma_{0}} W_{r}
$$

In the first example we take high-frequency wake field $\left(k \sigma_{0}=25, Q=1000, I=1000\right)$ and simulate damped synchrotron oscillations to the stable steady-state solution of Haissinski equation ${ }^{9}$

$$
\begin{aligned}
& \psi(x, p)=\frac{1}{\sqrt{2 \pi}} \exp \left(-\frac{p^{2}}{2}\right) \rho(x) \\
& \rho(x)=\rho_{0} \exp \left[-\frac{x^{2}}{2}+\int_{-\infty}^{x} d x^{\prime} \int_{-\infty}^{x^{\prime}} w\left(x^{\prime \prime}-x^{\prime}\right) \rho\left(x^{\prime \prime}\right) d x^{\prime \prime}\right]
\end{aligned}
$$

Two plots in Fig. 4 show the result. We chose initial bunch length to be two times lager than natural bunch length. Left plot shows the bunch distribution after the first synchrotron period. Right plot shows the bunch distribution after four damping times. In this example, the damping time is equal to 100 synchrotron periods. In each picture the upper curves show the emittance (red line), bunch length (violet line) and energy spread (green) in time (in synchrotron periods). We can see that approximately after two damping times the bunch distribution comes to the stable state. Left graphic on each plot shows the energy distribution and right - the particle distribution together with cavity RF 
voltage plus wake potential. Dotted lines show Haissinski solution. At the bottom of each plot, one can see the phase distribution function (left plot) and its colored projection (right plot). This test has shown that our algorithm works very well for steady state solutions, described by Haissinski equation.

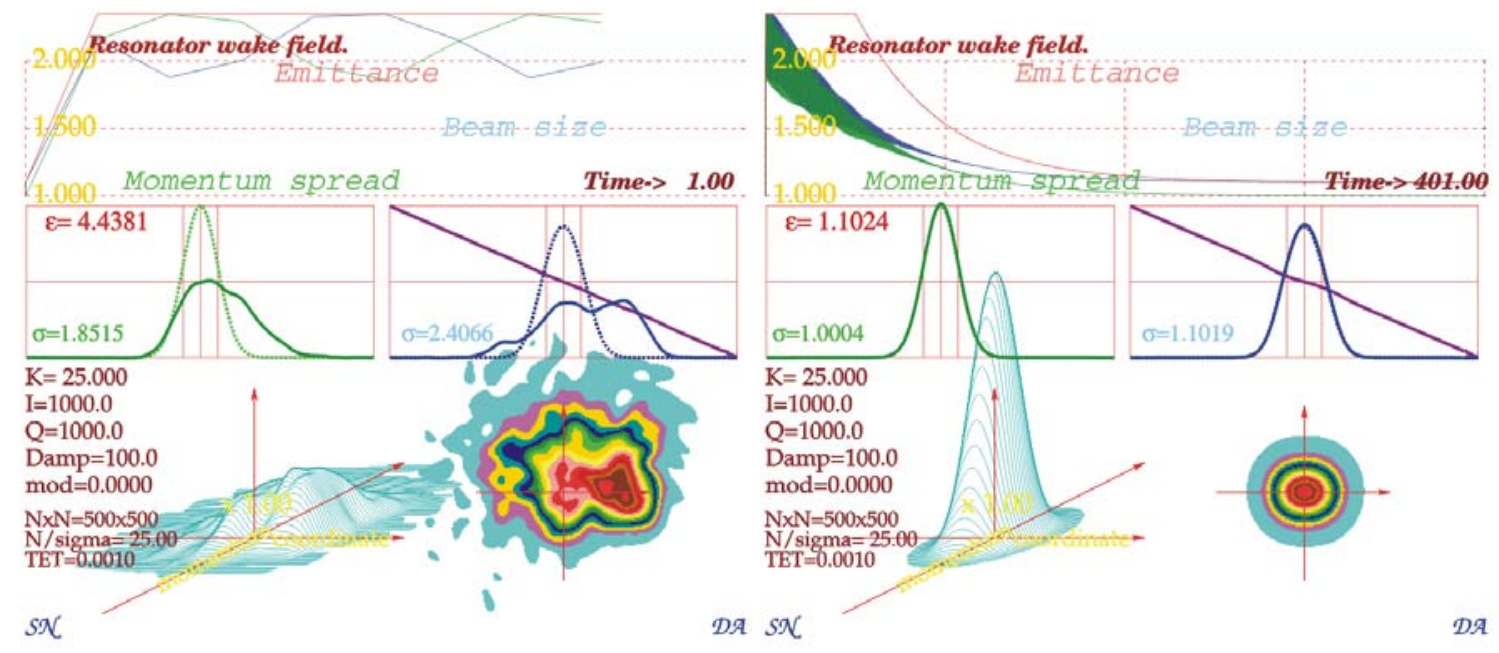

Figure 4: Bunch shape after the first synchrotron period (left plot) and steady state distribution after four damping times (right plot).

We also check algorithm for very unstable regime - saw-tooth instability. For this, we made comparison with the results of R. Baartman and M. D'Yachkov multiparticle tracking simulations ${ }^{10}$. They studied diffusion saw-tooth instability that happens for low-frequency wake field. We chose the following frequency $k \sigma_{0}=0.5$ and wake field parameters: $Q=1, I=30$ for comparison. When we applied these parameters to our algorithm, we found very good agreement with their results, with they showed in figure 1 and figure 4 in their paper ${ }^{10}$. Corresponding plots for this very interesting instability from our simulations are shown in Fig. 5. 

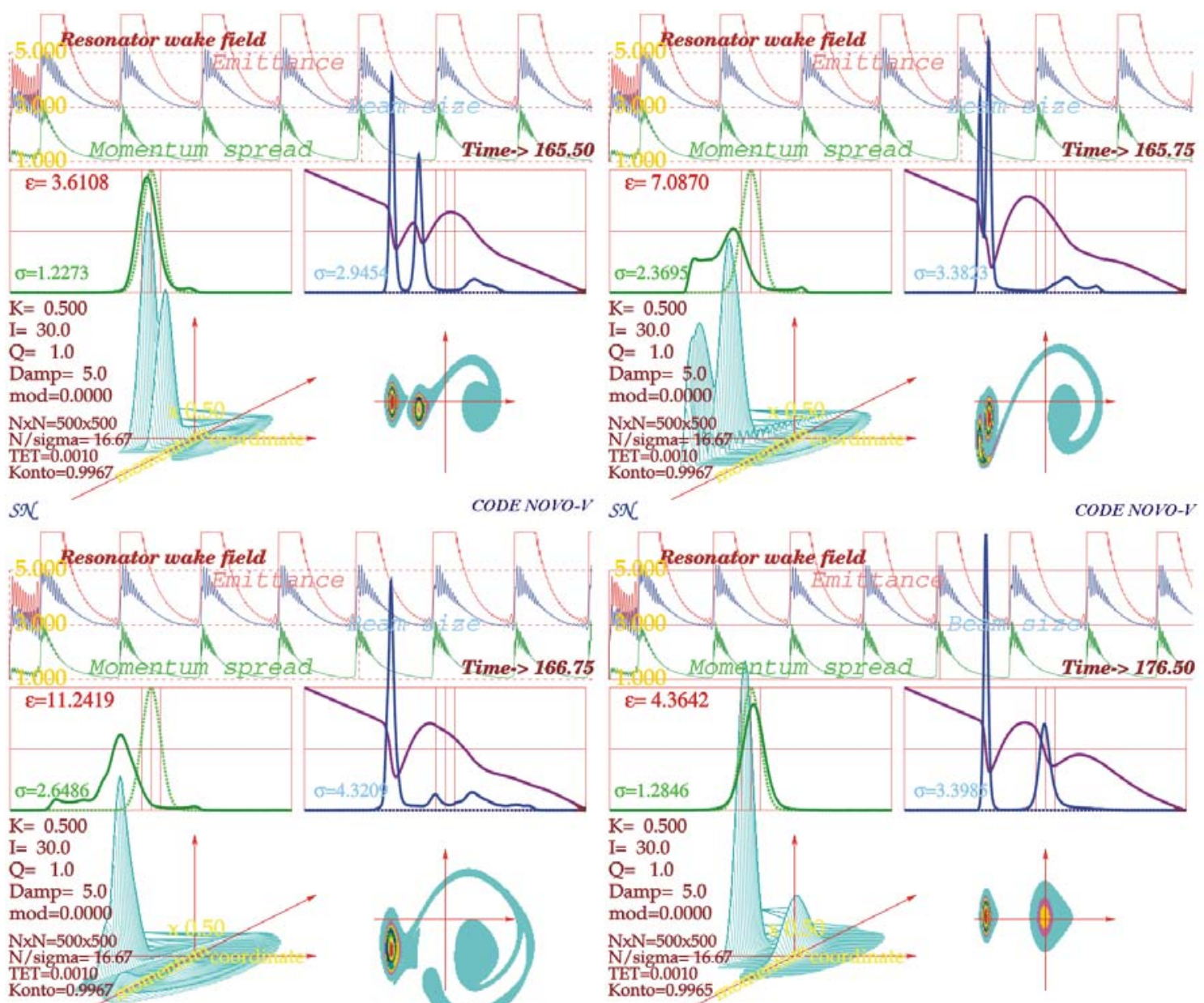

$S \mathcal{N}$

CODENOVO-V SK

CODE NOVO-V

Figure 5: Diffusion saw-tooth instability in four time snap-shots.

\section{APPLICATION}

We carried out instability study for the large frequency band of resonator wake field for different Q-number: 1 and 1000. Fig. 6 shows instability threshold as a function of frequency. Green line shows the value of intensity parameter, when"classical" microwave instability started. Red line with triangles and violet line with rhombs show the threshold for saw-tooth instability. Blue solid line with circles shows analytical estimation for threshold: $I_{t h r} \approx 1.8\left(k \sigma_{0}\right)^{2}$. We got this analytical quadratic behavior 
assuming that instability begins when the energy spread from the wake field becomes comparable to the RF focusing. We have found the coefficient for this relation from our simulations.

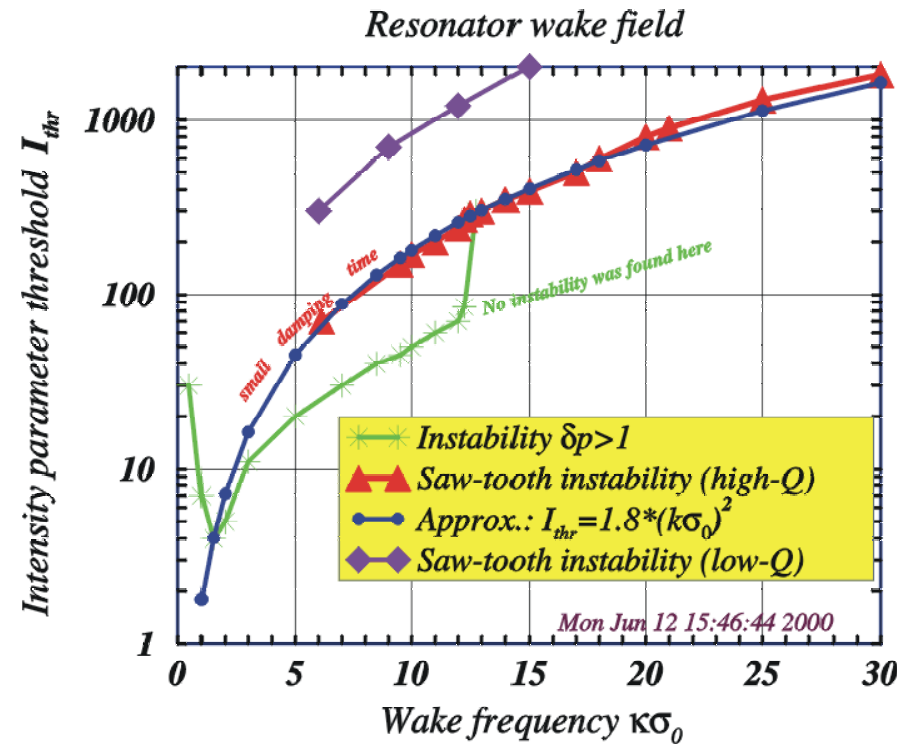

Figure 6: Single bunch instability thresholds for resonator wake field.

We found out that high frequency wake fields $\left(k \sigma_{0}>12\right)$ produce mainly saw-tooth instability. Fig. 7 shows an example of such instability.
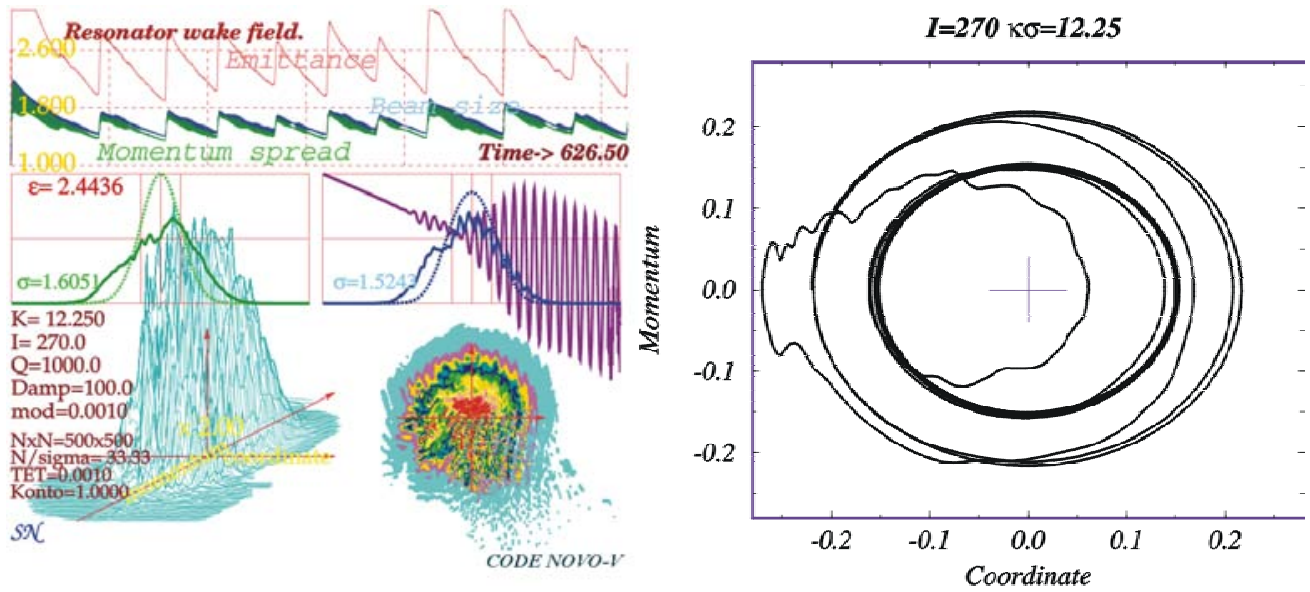

Figure 7: Bunch distribution during blow-up of high frequency saw-tooth instability (left plot).

Bunch center trajectory on the phase plane (right). 
It is interesting to note that the bunch center changes its orbit during the blow-up period in a quantum-like way: jumps from one orbit to another (right plot in Fig. 7).

The high efficiency of this algorithm allows detecting even a very weak instability. We take the steady-state solution of the Haissinski equation as initial condition for the bunch distribution and watch the bunch dynamics in time. One can presume that the bunch distribution is stable if it does not change in time. However, there is some weak instability, which blows up only after some period and nobody knows how long we have to wait for instability. However, we can easily find the instability, if we calculate the bunch power loss (energy loss of the bunch during one turn in the ring)

$$
\kappa(t)=\int_{-\infty}^{\infty} W(t, x) \rho(t, x) d x
$$
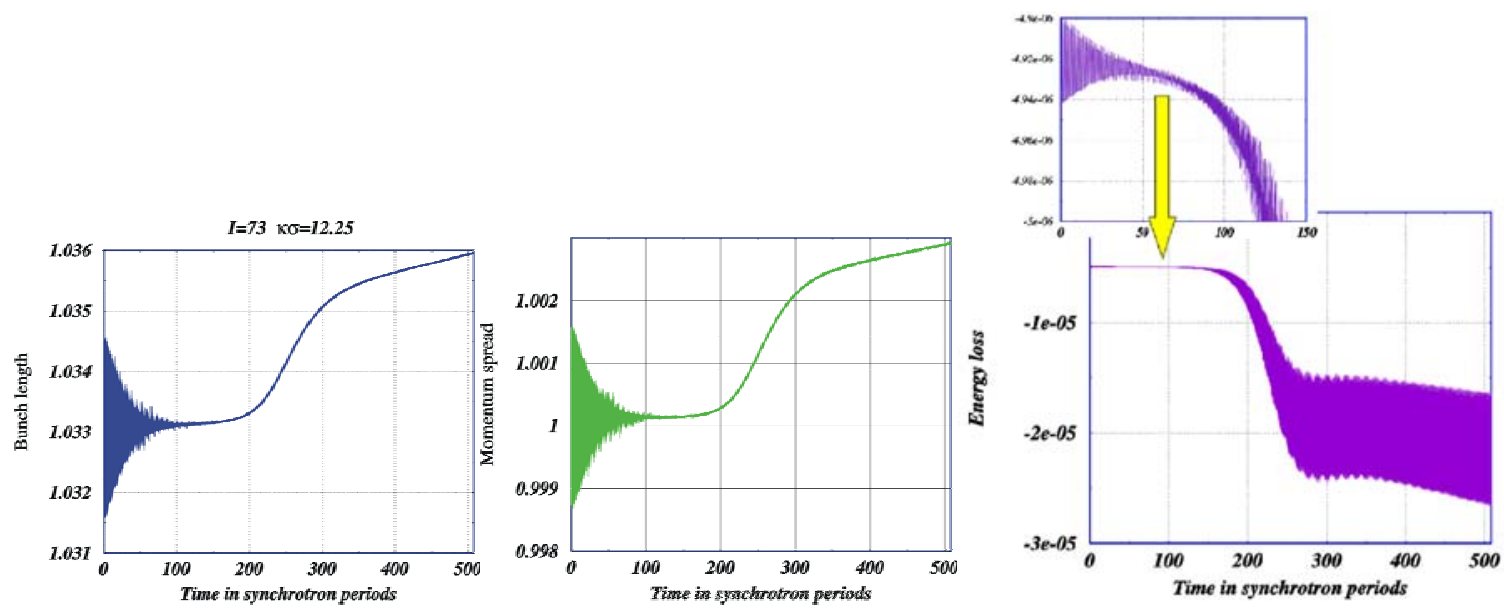

Figure 8: Weak instability. Bunch length (left plot), momentum spread (middle plot) and bunch energy loss (right plot) as functions of time.

Fig. 8 presents the example of the weak instability for the parameters: $k \sigma_{0}=12.5, Q=1000, I=73$. The bunch blows up only after 200 synchrotron periods 
and the change of the momentum spread and the bunch length is very small $(\sim 0.2-0.3$ $\%$ ). Bunch energy loss also increases at the blow up point; however, we can clearly see the instability development many periods before, starting after 70 synchrotron periods.

As a practical application of this algorithm, we show the results of the instability study for the Super-ACO machine. We use the impedance model suggested by G. Flynn $^{11}$. Main effect comes from sixteen circular pumping chambers, located between elliptical beam pipes. We assume that the pumping chambers can be described as "long" cavities, which we showed before in Fig. 1. The action of these chambers is so strong, that saw-tooth instability happens at the bunch current of $20 \mathrm{~mA}$. Left plot in Fig. 9 shows the bunch distribution for this kind of instability. It is interesting to note that the bunch length and momentum spread have small amplitude of oscillations.
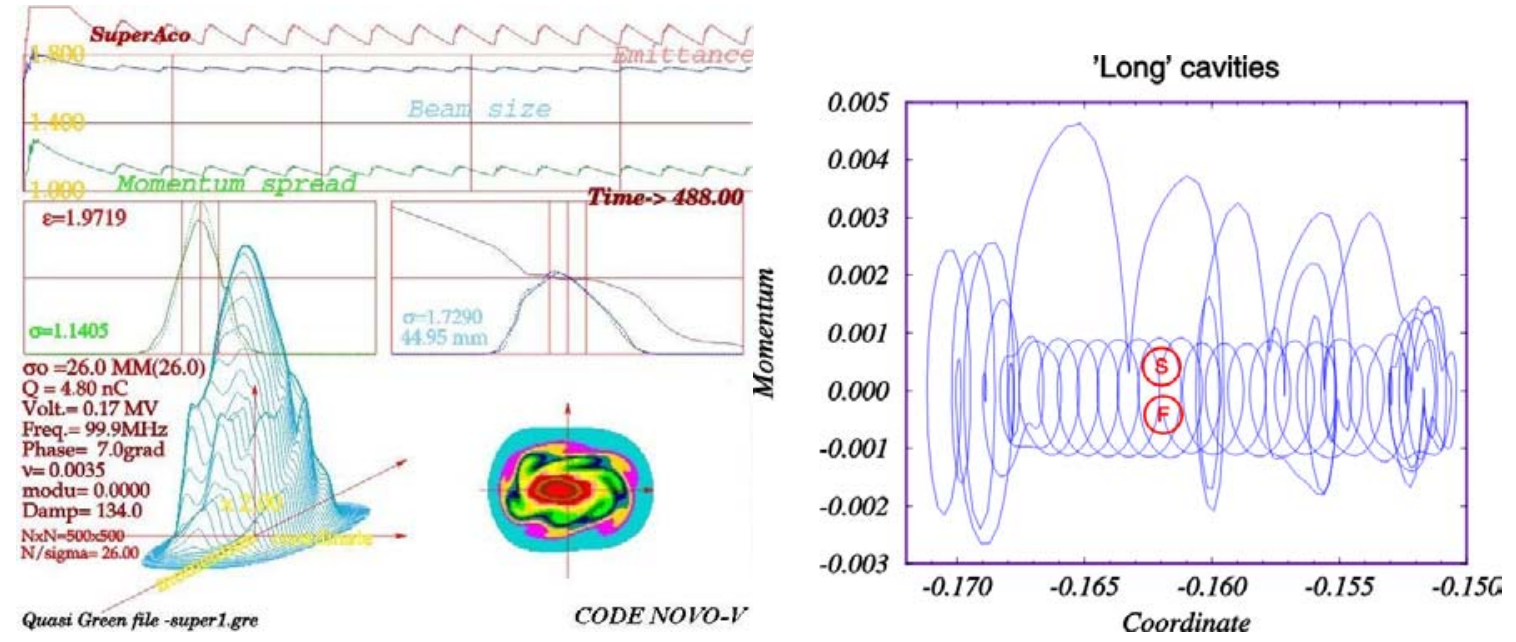

Figure 9: Saw-tooth instability for the bunch current of $20 \mathrm{~mA}$ (left plot). Right plot shows bunch centre trajectory on the phase plane.

Another application shows that eight small bellows (Fig. 10) can explain bursts of coherent radiation (left plot in Fig. 11) in VUV machine ${ }^{12}$, because of micro bunching in saw-tooth instability (right plot in Fig. 11). 


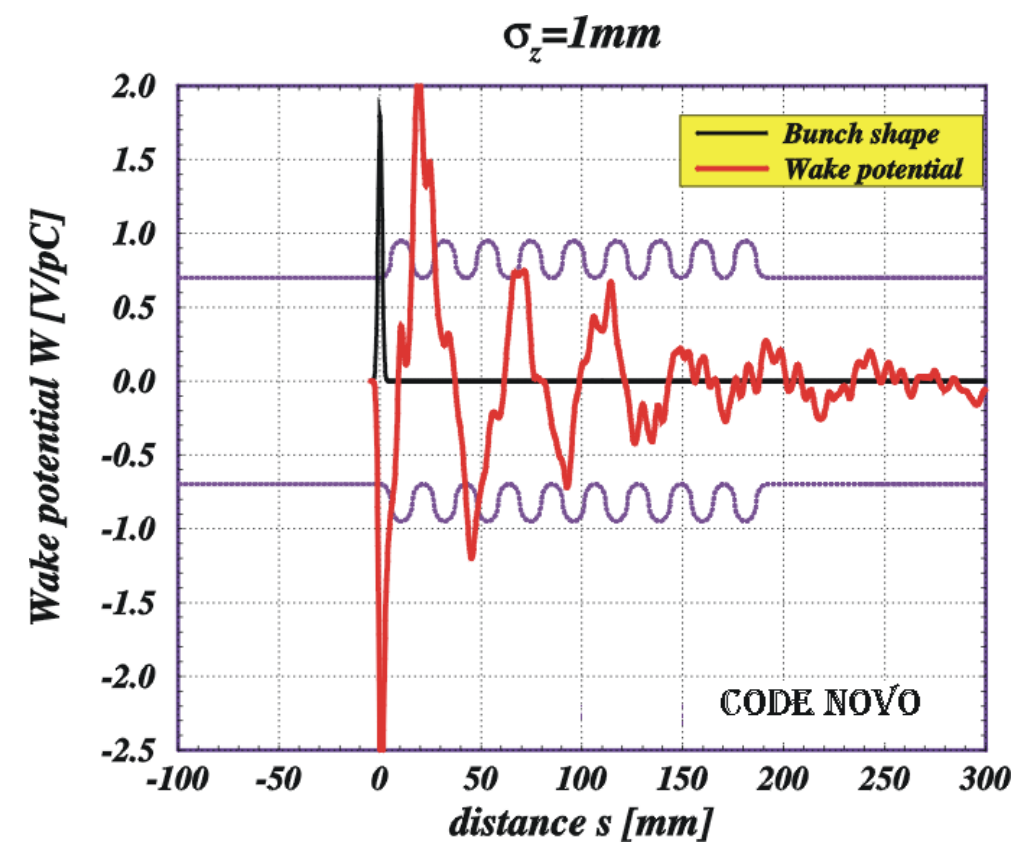

Figure 10: NSLS-VUV bellows shape (pink line) and wake potential of $1 \mathrm{~mm}$ bunch (red line). Black line shows bunch distribution.
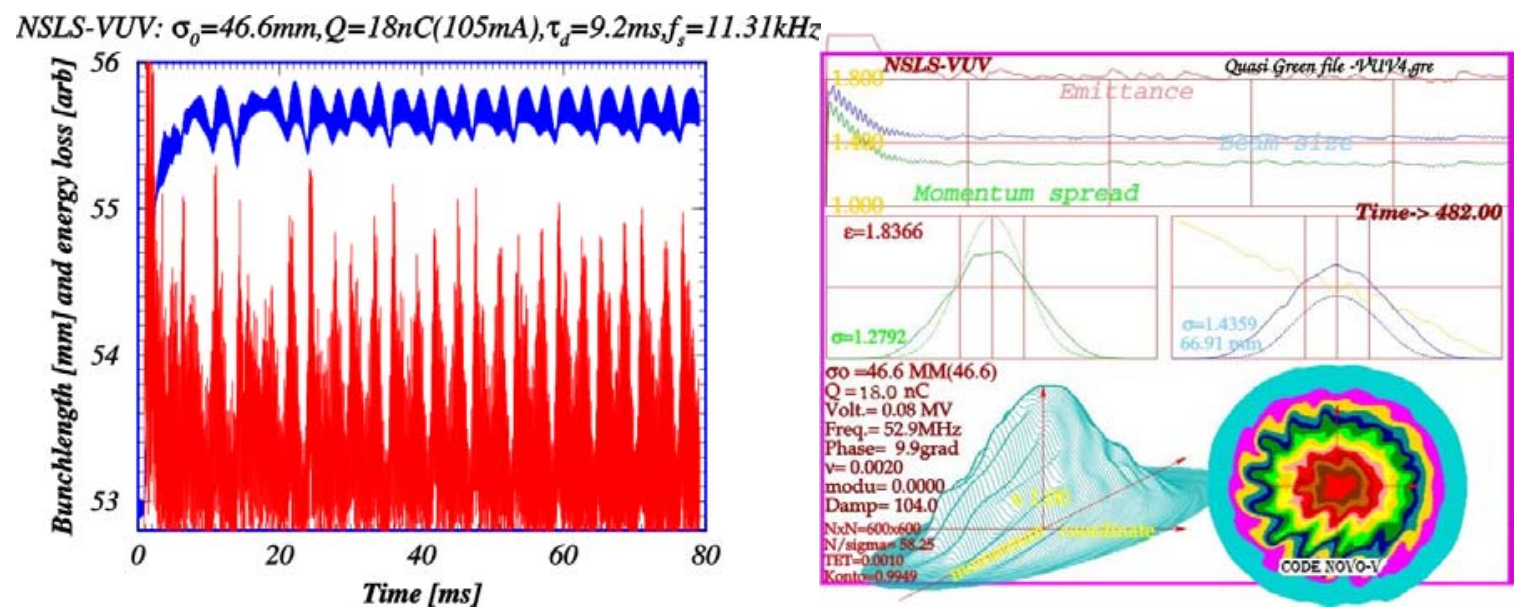

Figure 11. Left plot: Bunch length (blue line) and bunch energy loss (red line) as functions of time in saw-tooth instability. Right plot: Bunch distribution during a bust of wake field radiation.

Single bunch dynamics study for the PEP-II low energy ring (LER) showed that instability level is very high - more than $24 \mathrm{~mA}$ per bunch. This result was achieved for 
the wake potential that has been developed by S. Heifets ${ }^{13}$. Bunch lengthening and sawtooth instability above threshold current in LER are shown in Fig. 12.
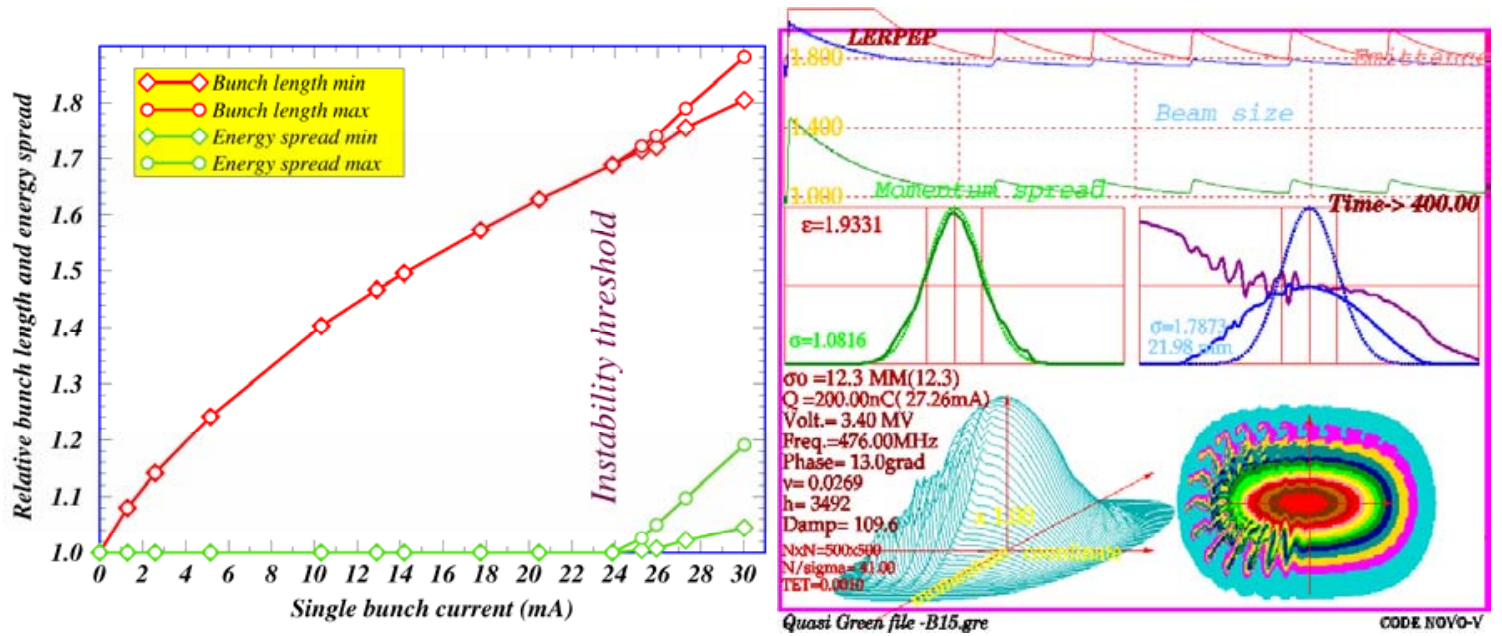

Figure 12: Bunch lengthening in the PEP-II LER (left plot). Right plot shows saw-tooth instability above threshold current.

The last example of application of the algorithm is devoted to the single bunch instability study for the NLC damping ring.
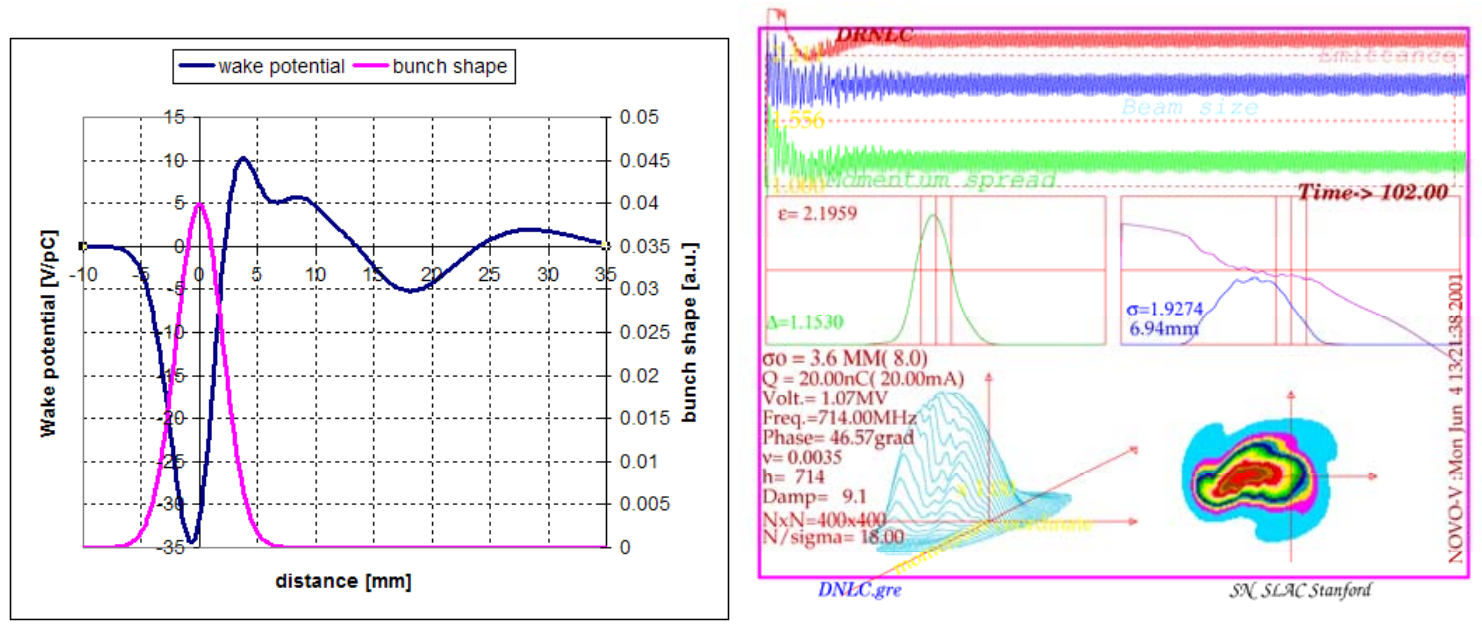

Figure 13. Left plot: Wake potential and bunch shape of $2 \mathrm{~mm}$ bunch. Right plot: Instability in DNLC at $20 \mathrm{~mA}$ bunch current. 
Wake potential has been developed by C.- $\mathrm{K} . \mathrm{Ng}^{14}$. The wake potential of $2 \mathrm{~mm}$ bunch is shown in the left plot in Fig. 13. Signs of instability begin to occur at approximately 20 $\mathrm{mA}$ per bunch, a factor of 8 greater than the charge for which the ring is being designed. Right plot in Fig. 13 shows the single bunch instability at the bunch current of $20 \mathrm{~mA}$. This instability has features of microwave instability.

\section{REFERENCE}

${ }^{1}$ A.Chao, Physics of Collective Beam Instabilities in High Energy Accelerators (John Wiley \& Sons, Inc., New York, 1993)

${ }^{2}$ A.Novokhatski, TESLA Meeting, Frascati, 1998, http://www.sis.lnf.it/talkshow/tesla98.htm

${ }^{3}$ A.Novokhatski, Workshop on Linear Colliders, LC'99, 1999, http://wwwsis.lnf.it/talkshow/lc99/Novokhatski/talk.pdf.

${ }^{4}$ A.Novokhatski and T, Weiland, EPAC'2000, p.

${ }^{5}$ B.Zotter and S.Kheifets, Impedances and Wakes in High Energy particle Accelerators (World Scientific, Singapore, 1998).

${ }^{6}$ K.Bane, K.Oide, PAC'95, 1995, p.245 (1984)

${ }^{7}$ M.Zobov et.al, International Workshop on Collective Effects and Impedance for B-factories, p. 110.

${ }^{8}$ A.Novokhatski, To be published

9 J.Haissinski, Nuovo Cimento 18B, p. 72 (1973).

${ }^{10}$ R.Baartman, M.D'Yachkov in PAC'95, 1995, p.3119.

${ }_{11}$ M.Billardon, et al, EPAC'98, 1998, p. 954.

${ }^{12}$ G.Carr et al, $P A C^{\prime} 99$, 1999, edited by A.Luccio, W.MacKay , p. 134.

${ }^{13}$ S. Heifets, private communication.

${ }^{14}$ J. Corlett et al, PAC'2001,Chicago, p. 1841 